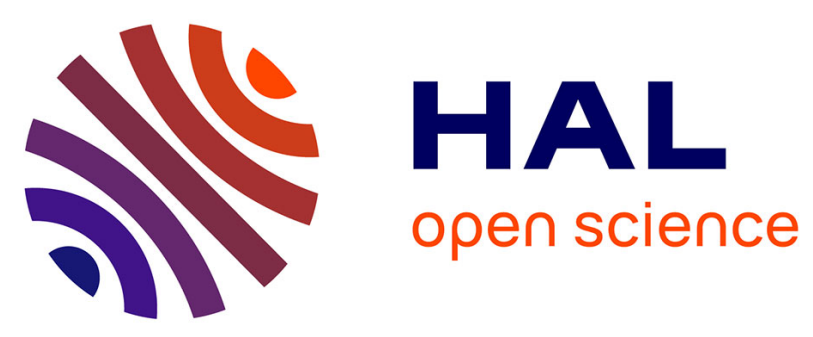

\title{
Measurements of comparative apparent thermal conductivity of large monolithic silica aerogels for transparent superinsulation applications
}

Arnaud Rigacci, Bruno Ladevie, Hébert Sallee, Bruno Chevalier, Patrick Achard, Olivier Fudym

\section{To cite this version:}

Arnaud Rigacci, Bruno Ladevie, Hébert Sallee, Bruno Chevalier, Patrick Achard, et al.. Measurements of comparative apparent thermal conductivity of large monolithic silica aerogels for transparent superinsulation applications. High Temperatures-High Pressures, 2002, 34 (5), pp.Pages 549-559. 10.1068/htjr065 . hal-00533820

\section{HAL Id: hal-00533820}

https: / hal-mines-paristech.archives-ouvertes.fr/hal-00533820

Submitted on 7 Nov 2019

HAL is a multi-disciplinary open access archive for the deposit and dissemination of scientific research documents, whether they are published or not. The documents may come from teaching and research institutions in France or abroad, or from public or private research centers.
L'archive ouverte pluridisciplinaire HAL, est destinée au dépôt et à la diffusion de documents scientifiques de niveau recherche, publiés ou non, émanant des établissements d'enseignement et de recherche français ou étrangers, des laboratoires publics ou privés. 


\title{
Measurements of comparative apparent thermal conductivity of large monolithic silica aerogels for transparent superinsulation applications
}

\author{
Arnaud Rigacci, Bruno Ladevie $\uparrow$, Hébert Sallee§, Bruno Chevalier§, Patrick Achard, \\ Olivier Fudym $\uparrow$ \\ École des Mines de Paris, Energetic Research Centre (CENERG), rue Claude Daunesse, BP 207, \\ F 06904 Sophia-Antipolis Cedex, France; fax: 334939575 35; e-mail: rigacci@cenerg.cma.fr; \\ - École des Mines d'Albi-Carmaux, Environmental and Energetic Research Centre, Route de Teilhet, \\ campus Jarlard, F 81000 Albi Cedex 09, France; § Centre Scientifique et Technique du Bâtiment \\ (CSTB), 24 rue Joseph Fourier, F 38400 Saint Martin d'Hères, France
}

\begin{abstract}
Large monolithic crack-free transparent silica aerogels were prepared by a patented sol-gel route, and then directly washed and dried with supercritical $\mathrm{CO}_{2}$. In order to characterise as precisely as possible the apparent thermal conductivity of such superinsulating materials, two methods were developed (so-called hot-band and micro-fluxmeter techniques) and the results obtained at room temperature and atmospheric pressure were compared with the standard hot-wire measurements under the same conditions. A reproducibility study showed no large deviations between the thermal conductivities obtained by these different techniques. This leads to the conclusion that aerogels prepared in this way present a good thermal and optical compromise (low thermal conductivity, high light transmission, and no significant radiative heat transport component at room temperature) and can be considered as transparent superinsulating materials.
\end{abstract}

\section{Nomenclature}

$\begin{array}{ll}A, B, C, D & \text { quadripole elements } \\ E & \text { extinction coefficient } \\ Q & \text { excitation heat flux } \\ R_{\mathrm{c}} & \text { constriction thermal resistance } \\ T & \text { temperature } \\ a & \text { thermal diffusivity } \\ x, y, z & \text { spatial coordinates } \\ p & \text { Laplace parameter } \\ t & \text { time }\end{array}$

$\begin{array}{ll}b, L & \text { lateral dimensions } \\ e & \text { thickness } \\ \lambda & \text { thermal conductivity } \\ \psi & \text { Laplace-Fourier flux } \\ \theta & \text { Laplace- Fourier temperature } \\ \rho & \text { density } \\ \rho c_{p} & \text { volumetric heat capacity } \\ \alpha_{n} & \text { Fourier parameter } \\ i & \text { index relating to the } i \text { th layer }\end{array}$

\section{Introduction}

Because of its particular internal porous texture, aerogel material presents numerous interesting potential applications such as catalyst support, supercapacitor, sound absorber, and (transparent or opaque) highly insulating material (Hrubesch 1998). At present there is an increasing industrial interest in aerogels, particularly in the thermal insulation and electric storage fields (Biesmans et al 1998; Schmidt and Schwertfeger 1998).

In this class of materials, silica aerogel combines optical transparency in the visible range and good thermal insulation properties. This is the reason why one of the main domains of its potential applications is in double glazing to replace the air layer between the glass panels (Wittwer 1992). Its low apparent thermal conductivity associated with its opacity towards infrared radiation at temperatures close to room temperature may make it possible to improve the insulation properties of classic double glazing without significantly restricting optical transparency.

For such an application, it is important to determine as precisely as possible the thermal properties of the spacer material. Designing an experimental device to estimate 
the thermophysical transport properties of such a superinsulating material as silica aerogel is generally difficult. This is the reason why in this study we compare four different methods to determine the possible influence of the thermal characterisation procedure.

Apparent thermal conductivity was first measured by an extended hot-wire method, because this standard method (Carslaw and Jaeger 1959) is currently being employed for thermal characterisation of aerogels (Ebert et al 1993; Zeng et al 1994). This technique is easy to use but, even if the cylindrical semi-infinite medium assumption avoids the problem of considering heat losses at the ends of the medium, some heat loss effects are possible at the ends of the wire. Such axial or radial heat losses can be estimated (Healy et al 1976).

The use of the transient flash method (Degiovanni 1977) for measuring the thermal diffusivity of silica aerogel is difficult because of heat losses around the sample. Martin et al (1994) tried to improve the setup by adding two metal plates on each side of the sample. But this makes the experiment more complicated and lateral heat losses are not completely eliminated.

For this reason, a special device was developed (Ladevie 1998). The hot-band method proposed here tries to combine all advantages of previous methods. The main idea is to control the spread of the heat flux inside the insulating sample by adding a highly conductive metal support, so as to eliminate lateral heat losses. A probe similar to the one employed in the hot-wire system is used at a single time-dependent temperature with a planar heating device. A two-dimensional transient model has been developed. The results obtained by this innovative method were confirmed by thermal diffusivity measurement with the flash method (Gobbe et al 1988).

Finally, to analyse the validity of these transient measurements, a steady-state method was developed which was mainly based on the NF ISO 8301 standard (1991) in the twofluxmeter configuration. This technique also permitted us to roughly estimate the radiative contribution to heat transport close to room temperature.

From the materials point of view, it is important to note that the samples characterised in the present paper were dried by an interesting recent method based on washing the wet gels directly under a flow of supercritical $\mathrm{CO}_{2}$ (Van Bommel and de Haan 1994), and not under a flow of liquid $\mathrm{CO}_{2}$ (Tewari et al 1986).

The studies presented in this paper were performed as part of a collaborative projectthe 'PACTE ADEME' (Agence de l'Environnement et de la Maîtrise de l'Énergie) -involving several partners, coming both from universities and the industry.

\section{Aerogel preparation}

\subsection{Synthesis of wet gel}

The gelation occurred through a double-catalytic sol-gel process described in detail by Pajonk et al (1995). The first step consisted in substoichiometric hydrolysis of tetraethoxysilane (TEOS) molecules with catalysis by an acid $\left(\mathrm{H}_{2} \mathrm{SO}_{4}\right)$ in ethanol at $35^{\circ} \mathrm{C}$. Such prepolymerised precursors are called polyethoxydisiloxanes (PEDS-Px) and are synthesised by PCAS (BP 11, F 01161 Longjumeau, France). During the second step, the obtained chains of polyethoxydisiloxane reacted together under hydrofluoric acid (HF) (21 $\mathrm{N}$ and a constant volume fraction equal to 0.02) in ethylacetoacetate (ETAC), with a 4 min slow stirring at room temperature. The gels thus synthesised were aged in a pure ethylacetoacetate bath for $72 \mathrm{~h}$.

This study is focused on one family of wet gels synthesised with a prepolymerised precursor with a silica content of $28 \mathrm{wt} \%$, a pre-hydrolysis rate (water molecules per TEOS molecules) of 1.2, and a volume fraction of polyethoxydisiloxane in ethylacetoacetate equal to 0.5 . 


\subsection{Supercritical drying of wet gel}

The plate gels $\left(13 \times 13 \times 1 \mathrm{~cm}^{3}\right)$ were horizontally placed in a pre-heated autoclave so that their whole surface was homogeneously in contact with the surrounding phase. They were then washed under a dynamic direct supercritical $\mathrm{CO}_{2}$ flow at constant temperature, pressure, and mass flow rate. When the total amount of ethylacetoacetate was recovered, and was replaced in the porous structure by supercritical $\mathrm{CO}_{2}$, the system was isolated and the supercritical $\mathrm{CO}_{2}$ was slowly released to avoid the occurrence of depressurisation stresses due to the low permeability of the gels (Scherer 1994). During this drying phase no gel shrinkage was observed. This is mainly due to the blockage of the polycondensation reactions in supercritical $\mathrm{CO}_{2}$, the decrease of the capillary forces, and the slow depressurisation rate.

Such a reproducible process permitted us to obtain large flat and crack-free monolithic aerogels with an average density closely centred around $0.160 \pm 0.010 \mathrm{~g} \mathrm{~cm}^{-3}$. We studied the thermal properties of four sets of aerogels, coming from four different direct supercritical extractions.

\section{Measurements of apparent thermal conductivity: experimental setup and methods}

\subsection{The extended hot-wire method}

The apparent thermal conductivity was first measured with an apparatus developed by CSTB Grenoble, called the CT-Meter, based on the classic hot-wire method (Quenard and Sallee 1991). The scheme of the experimental setup is shown in figure 1.

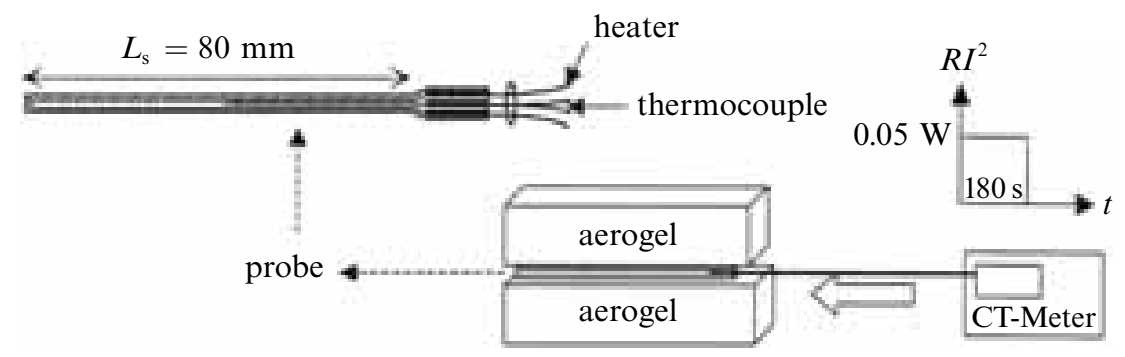

Figure 1. Scheme of the hot-wire device.

As the theoretical and experimental principles of this method are well known; we shall therefore only briefly outline here the procedure.

At room temperature, for all the measurements, a step power $(0.05 \mathrm{~W}$ during $180 \mathrm{~s})$ is applied to the probe, placed between two identical samples. The evolution of the probe temperature in time is measured with a thermocouple. The apparent thermal conductivity, $\lambda_{\text {eq }}$, is then classically obtained from the experimental slope of the thermogram:

$$
\Delta T=\frac{R I^{2}}{L_{\mathrm{s}}} \frac{1}{4 \pi \lambda} \ln (t)+\frac{R I^{2}}{L_{\mathrm{s}}} \frac{1}{4 \pi \lambda} \ln \left(\frac{4 a}{1.781 R^{2}}\right)
$$

where $R$ and $L_{\mathrm{s}}$ are the resistance and the length of the probe, $I$ is the intensity of the power peak, and $a$ is thermal diffusivity.

The probe used consisted of a Constantan heater and a T-type thermocouple cut in a $3 \mathrm{~mm}$ Kapton support. The track width and length were respectively $25 \mu \mathrm{m}$ and $250 \mu \mathrm{m}$.

In this study, only thick aerogel samples were characterised by this method. Some experimental surface observations with an infrared camera had previously shown that with a measurement time of $180 \mathrm{~s}$ and with the specific heat of the prepared aerogels the hypothesis of a semi-infinite medium applied only to samples with a thickness greater than $1 \mathrm{~cm}$ (Rigacci 1998). 


\subsection{The hot-band method}

Although this method has been recently described in detail (Ladevie et al 1999), we shall outline here the main principles of the experimental device described in figure 2.

The heating probe is made of two thin-foil resistances (Minco France, F 09310 Aston, France) in which a K-type thermocouple is inserted to measure the variation of the temperature of the probe with time. An electric generator supplies a step-power excitation to the probe. The thermocouple signal is recorded with a digital oscilloscope. The only precaution as far as the samples are concerned is with respect to their size, parallelism, and symmetry. A $10 \mathrm{~cm}$ thick brass cylinder is used as a conducting semiinfinite medium.

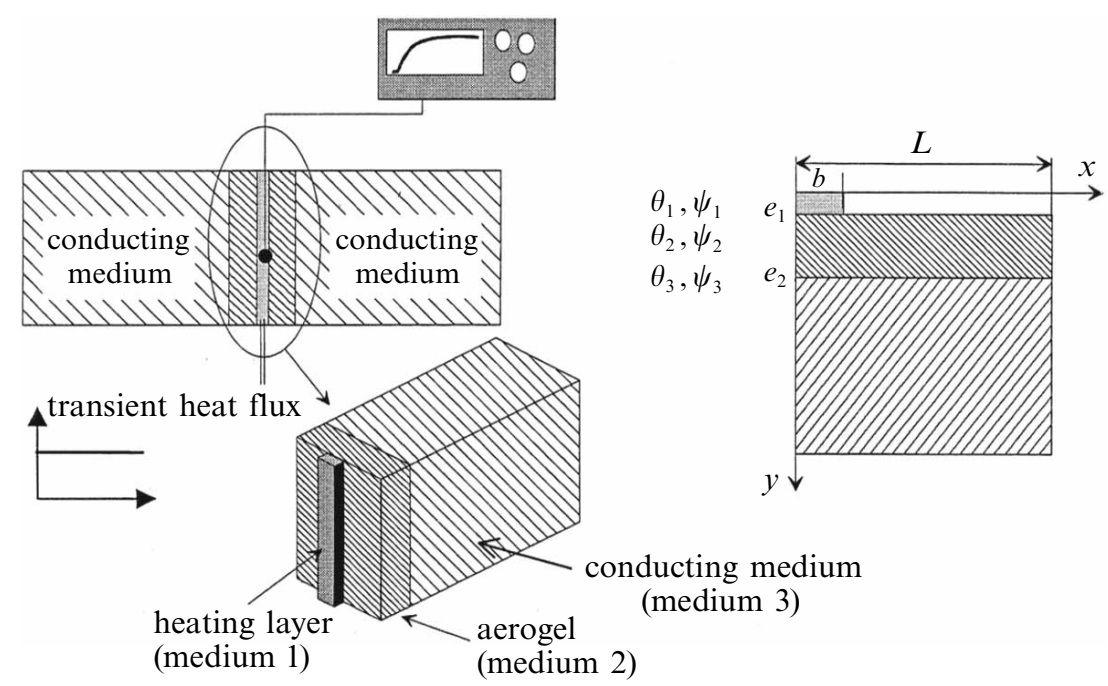

Figure 2. Scheme and overview of the geometrical parameters of the hot-band device.

3.2.1 Transfer inside the heating layer (medium 1). This layer is metallic and is considered to be infinitely thin. Thus, temperature distribution is assumed to be uniform in the $z$-direction. This yields then:

$$
\left(\rho c_{p}\right)_{1} e_{1} \frac{\partial T_{1}^{*}}{\partial t}=Q+\Phi_{2}\left(y=e_{1}\right)
$$

where $Q$ is the Joule-effect heat flux and $\Phi_{2}\left(y=e_{1}\right)$ describes the heat flux penetrating inside the insulating sample (medium 2).

3.2.2 Transfer inside the insulating sample (medium 2) and inside the conducting medium (medium 3). Heat transfer is here assumed to be purely conductive and the sample isotropic. This yields:

$$
\frac{\partial^{2} T_{i}^{*}}{\partial x^{2}}+\frac{\partial^{2} T_{i}^{*}}{\partial y^{2}}=\frac{1}{a_{i}} \frac{\partial T_{i}^{*}}{\partial t}, \quad \text { with } i=2,3,
$$

with transformed flux and temperature continuity at interfaces between medium 1 and medium 2, and medium 2 and medium 3.

Initially, the whole system is assumed to be at a uniform temperature $T_{0}$. A new variable is then introduced: $T_{i}=T_{i}^{*}-T_{0}$. To describe the previous system in a less complex form, Laplace and Fourier transforms are applied. The previous equation then becomes an ordinary differential equation

$$
\frac{\mathrm{d}^{2} \theta_{i}}{\mathrm{~d} y^{2}}=\left(\frac{p}{a_{i}}+\alpha_{n}^{2}\right) \theta_{i}, \quad \text { with } \alpha_{n}^{2}=\frac{n \pi}{L},
$$


with heat flux defined as follows:

$$
\psi_{i}\left(\alpha_{n}, p, y\right)=-\lambda_{i} \frac{\mathrm{d} \theta_{i}\left(\alpha_{n}, p, y\right)}{\mathrm{d} y} .
$$

Using the quadrupole presentation (Batsale et al 1994), we obtain a simple relationship between the transformed temperature and the flux vector at boundaries of each medium:

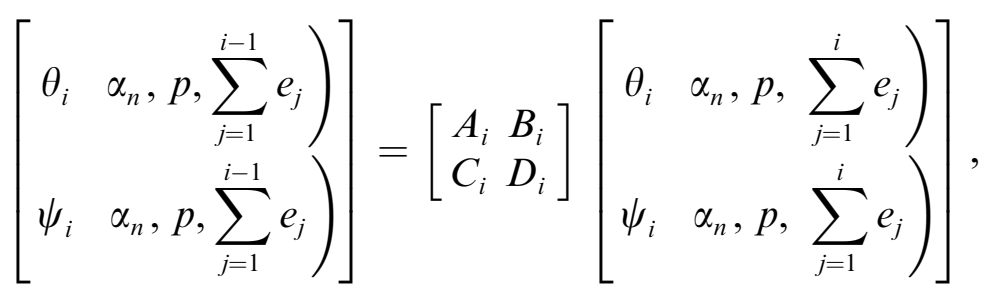

with

$$
\begin{aligned}
& A_{i}=D_{i}=\cosh \left(K_{i} e_{i}\right), \quad B_{i}=\frac{\sinh \left(K_{i} e_{i}\right)}{\lambda_{i} K_{i}} \\
& C_{i}=\lambda_{i} K_{i} \sinh \left(K_{i} e_{i}\right), \quad \text { and } K_{i}=\left(\frac{p}{a_{i}}+\alpha_{n}^{2}\right)^{1 / 2} .
\end{aligned}
$$

Medium 3 is considered as semi-infinite so that the transformed temperature distribution is in the form:

$$
\theta_{3}\left(\alpha_{n}, p, y\right)=\theta_{3}\left(\alpha_{n}, p, e_{1}+e_{2}\right) \exp \left(-K_{3}\right) y .
$$

This yields:

$$
\theta_{3}\left(\alpha_{n}, p, y\right)=\frac{1}{\lambda_{3} K_{3}} \exp \left(-K_{3} y\right)
$$

The entire system can be described in transformed space as:

$$
\left[\begin{array}{l}
\theta_{1}\left(\alpha_{n}, p, 0\right) \\
\Psi_{1}\left(\alpha_{n}, p, 0\right)
\end{array}\right]=\left[\begin{array}{ll}
1 & 0 \\
\left(\rho c_{p}\right)_{1} e_{1} p & 1
\end{array}\right]\left[\begin{array}{ll}
A_{2} & B_{2} \\
C_{2} & D_{2}
\end{array}\right]\left[\begin{array}{l}
\theta_{3}\left(\alpha_{n}, p, e_{1}+e_{2}\right) \\
\Psi_{3}=\lambda_{3} K_{3} \theta_{3}\left(\alpha_{n}, p, e_{1}+e_{2}\right)
\end{array}\right] .
$$

The transformed temperature measured on the heating plate is then

$$
\theta_{1}\left(\alpha_{n}, p, 0\right)=\frac{\left\{A_{2}+B_{2} \lambda_{3} K_{3}\right\} \psi_{1}\left(\alpha_{n}, p, 0\right)}{C_{2}+A_{2}\left(\rho c_{p}\right)_{1} e_{1} p+\lambda_{3} K_{3}\left\{B_{2}\left(\rho c_{p}\right)_{1} e_{1} p+D_{2}\right\}} .
$$

This expression is rather complex and can be inverted in real space by numerical computation. Nevertheless, some asymptotic expansions can give some insights to the physical behaviour of the system.

\subsubsection{Physical behaviour of the system by asymptotic assumption.}

(a) Asymptotic behaviour of expression (10) when $\left(\rho c_{p}\right)_{1} e_{1}=0$ and $\lambda_{3} \rightarrow \infty$.

Expression (10) then yields:

$$
\theta_{1}\left(\alpha_{n}, p, 0\right)=\frac{\tanh \left(\alpha_{n} e_{2}\right)}{\lambda_{2} \alpha_{n}} \frac{Q b \sin \left(\alpha_{n} b\right)}{p \alpha_{n}},
$$

with $e_{2}$, the thickness of the sample (figure 2) and $\alpha_{n}$, the Fourier parameter $\left(\mathrm{m}^{-1}\right)$ as defined in equation (4).

In real space, the expression for the temperature measured at the centre of the plate is:

$$
T(0,0, t)=\frac{e_{2} Q b}{\lambda_{2} L}+\frac{2 Q b}{\lambda_{2} L} \sum_{n=1}^{\infty} \frac{\tanh \left(\alpha_{n} e_{2}\right)}{b \alpha_{n}} \frac{\sin \left(\alpha_{n} b\right)}{\alpha_{n}}=R_{\mathrm{c}} Q
$$


where $R_{\mathrm{c}}$ is the constriction resistance between the heating plate and the semi-infinite cool plate. The assumption $\lambda_{3} \rightarrow \infty$ is here equivalent to the Dirichlet zero-temperature condition at the depth $y=e_{1}+e_{2}+e_{3}$.

(b) Asymptotic behaviour of expression (10) when $\left(\rho c_{p}\right)_{1} e_{1}=0$ and $\left(\rho c_{p}\right)_{2} e_{2}=0$.

We have then:

$$
\theta_{1}\left(\alpha_{n}, p, 0\right)=\frac{1}{\left[\lambda_{3}\left(\rho c_{p}\right)_{3} p\right]^{1 / 2}} \frac{Q b \sin \left(\alpha_{n} b\right)}{p \alpha_{n}}+\frac{\tanh \left(\alpha_{n} e_{2}\right)}{\lambda_{2} \alpha_{n}} \frac{Q b \sin \left(\alpha_{n} b\right)}{p \alpha_{n}} .
$$

In real space, the temperature measured at the centre of the plate is:

$$
T(0,0, t)=\frac{Q b}{L \pi^{1 / 2}\left[\lambda_{3}\left(\rho c_{p}\right)_{3}\right]^{1 / 2}} t^{1 / 2}+R_{\mathrm{c}} Q .
$$

An approximate expression such as equation (14) helps to understand the variation of temperature with time: $T(x=0, y=0, t)$. The first term depends only on the properties of medium 3. The second term (constant) depends only on thermal conductivity $\lambda_{2}$ and geometrical parameters.

A first simple estimation method is as follows:

(i) estimation of $R_{\mathrm{c}} Q$ at the ordinate origin (extrapolated),

(ii) fixing the value of $\left(\rho c_{p}\right)_{2}$ at $\left(\rho c_{p}\right)_{1}$ to begin the numerical estimation.

Since the Joule-effect energy is estimated by electrical measurement of the heating resistance, estimation of effusivity $\left[\lambda_{3}\left(\rho c_{p}\right)_{3}\right]^{1 / 2}$ of medium 3 is a good way to verify the conservation of the heat flow inside the system.

This independence of thermophysical properties of medium 2 and medium 3 can constitute the basic step for implementing a classical numerical estimation method which minimises the difference between experimental values and the exact expression (10) (Beck 1977). We have used a Nelder Mead minimisation algorithm (Press et al 1986).

\subsection{The micro-fluxmeter method}

The aerogel is placed between two isothermal plates kept at two different constant temperatures $\left(T_{\mathrm{f}}\right.$ and $\left.T_{\mathrm{c}}\right)$ by continuous fluid circulation. The thermal flux through the sample thickness $e$ is measured by two receivers closely stuck to the sample (figure 3 ). A spring system maintains a good contact between the sample and the plates.

Each isothermal surface contains a very sensitive thermal fluxmeter which makes it possible to follow the variation of the fluxes with time (both $\Phi_{\mathrm{f}}$ and $\Phi_{\mathrm{c}}$ ) during establishment of thermal equilibrium:

$$
\begin{aligned}
& \Phi_{\mathrm{c}}=\Phi_{(0, t)}=\lambda \frac{\Delta T}{e}\left[1+2 \exp \left(-\frac{a \pi^{2}}{e^{2}} t\right)+2 \exp \left(-\frac{4 a \pi^{2}}{e^{2}} t\right)\right], \\
& \Phi_{\mathrm{f}}=\Phi_{(e, t)}=\lambda \frac{\Delta T}{e}\left[1-2 \exp \left(-\frac{a \pi^{2}}{e^{2}} t\right)+2 \exp \left(-\frac{4 a \pi^{2}}{e^{2}} t\right)\right], \\
& \sum \Phi=\Phi_{\mathrm{c}}+\Phi_{\mathrm{f}}=2 \lambda \frac{\Delta T}{e}\left[1+2 \exp \left(-\frac{4 a \pi^{2}}{e^{2}} t\right)\right],
\end{aligned}
$$

with $\Delta T=T_{\mathrm{c}}-T_{\mathrm{f}}$. Near thermal equilibrium, the fluxes converge and the sum of the fluxes becomes constant. Over long times, the exponential term can be neglected and the apparent thermal conductivity can be obtained from the sum according to the following simple formula:

$$
\lambda=\frac{e}{\Delta T} \times \frac{1}{2} \sum \Phi .
$$




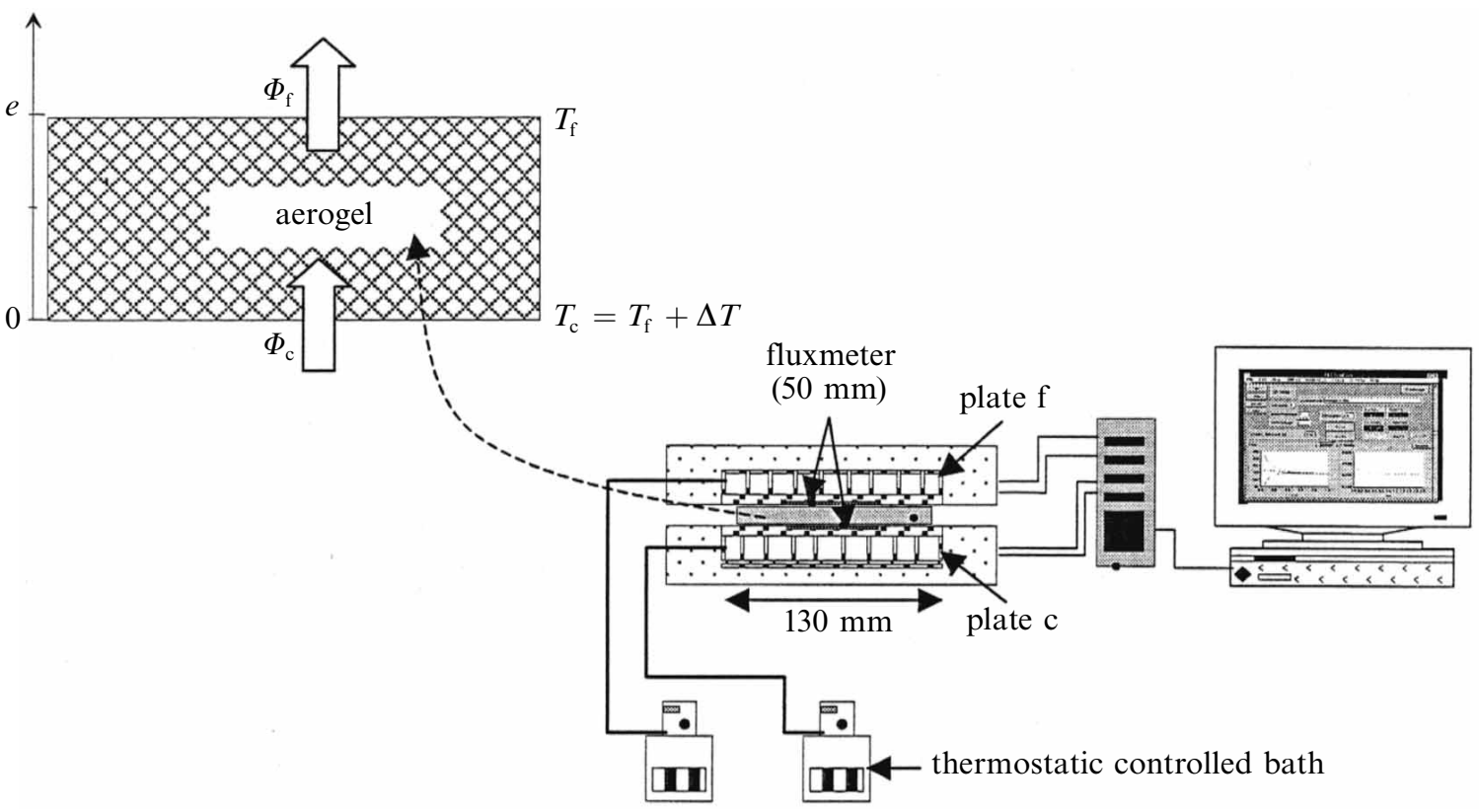

Figure 3. Scheme of the micro-fluxmeter device.

On the assumption that the thermal equilibrium is reached at $t=0$, this sum method allows us to obtain a convergence four times faster than the single-flux method, as with the recent Holometrix apparatus (figure 4).

From the accuracy point of view, it is important to note that the fluxmeters used at present, developed by the American firm Vattel, are known to have a very high sensitivity $\left(470 \mathrm{mV} \mathrm{W}^{-1} \mathrm{~cm}^{-2}\right)$, and consequently are very suitable for thermal characterisation of such insulating materials as silica aerogel. But before they are used, they need to be calibrated. Experimental fluxes observed during aerogel characterisation were between $4 \times 10^{-3}$ and $16 \times 10^{-3} \mathrm{~W} \mathrm{~cm}^{-2}$, even though the calibration coefficient provided by the manufacturer was for calibrating thermal fluxes of $0.197 \mathrm{~W} \mathrm{~cm}^{-2}$ (which represents a flux through each $5 \times 5 \mathrm{~cm}^{2}$ receiver close to $5 \mathrm{~W}$ ). Each fluxmeter is then directly calibrated with a reference material (a mineral fibre board) with a known thermal conductivity of $34.5 \pm 0.2 \mathrm{~mW} \mathrm{~m}^{-1} \mathrm{~K}^{-1}$ and three different thicknesses. The performed polynomial regressions gave the following flux/voltage calibration curves:

Receiver 1: $\Phi_{\mathrm{f}} / \mathrm{W} \mathrm{m}^{-2}=-0.21+195.1(\mathrm{~V} / \mathrm{mV})-9.92(\mathrm{~V} / \mathrm{mV})^{2}$,

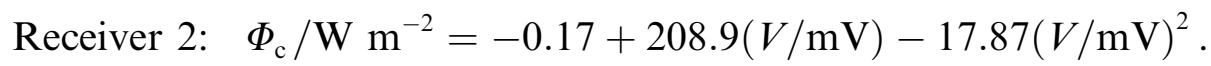

The apparent thermal conductivities obtained by this global method are estimated to have an experimental uncertainty close to $\pm 0.5 \mathrm{~mW} \mathrm{~m}^{-1} \mathrm{~K}^{-1}$.

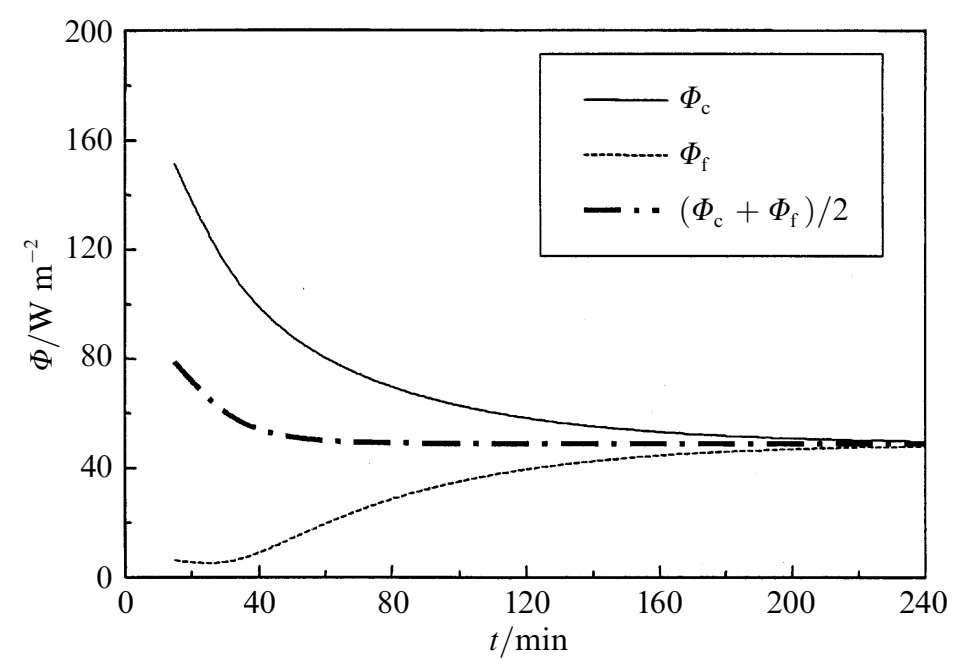

Figure 4. Flux convergence observed with the following thermophysical properties: $\rho=0.160 \pm 0.010 \mathrm{~g} \mathrm{~cm}^{-3}$; $c_{p}=0.9 \mathrm{~J} \mathrm{~g}^{-1} \mathrm{~K}^{-1} ; \Delta T=35 \mathrm{~K}$. 


\section{Results}

\subsection{Comparison}

All the thermal results of this study were obtained in air at atmospheric pressure. The corresponding average values are listed in the following tables. Those values and the associated deviations come from a reproducibility study based on five measurements per sample in each aerogel set.

Table 1. Hot-wire results at atmospheric pressure $\left(T_{\mathrm{m}}\right.$ represents the average measurement temperature, $\rho=0.160 \pm 0.010 \mathrm{~g} \mathrm{~cm}^{-3}$ ).

\begin{tabular}{lrll}
\hline Sample set & \multicolumn{1}{c}{$e / \mathrm{mm}$} & $T_{\mathrm{m}} / \mathrm{K}$ & $\lambda / 10^{-3} \mathrm{~W} \mathrm{~m}^{-1} \mathrm{~K}^{-1}$ \\
\hline SC1 & $9.0 \pm 0.1$ & $301 \pm 0.2$ & $18.0 \pm 0.5$ \\
SC5 & $11.0 \pm 0.1$ & $199 \pm 0.2$ & $19.0 \pm 0.5$
\end{tabular}

Table 2. Hot-band results at atmospheric pressure $\left(a, \rho c_{p}\right.$, and $T_{0}$, respectively, represent thermal diffusivity, volume specific heat, and initial temperature; $\rho=0.160 \pm 0.010 \mathrm{~g} \mathrm{~cm}^{-3}$ ).

\begin{tabular}{llllll}
\hline Sample set & $e / \mathrm{mm}$ & $T_{0} / \mathrm{K}$ & $\rho c_{p} / 10^{5} \mathrm{~J} \mathrm{~m}^{-3} \mathrm{~K}^{-1}$ & $\lambda / 10^{-3} \mathrm{~W} \mathrm{~m}^{-1} \mathrm{~K}^{-1}$ & $a / 10^{-8} \mathrm{~m} \mathrm{~s}^{-2}$ \\
\hline SC5 & $4.3 \pm 0.1$ & $295 \pm 0.2$ & $1.45 \pm 0.15$ & $19.7 \pm 0.3$ & $13.5 \pm 0.5$
\end{tabular}

Table 3. Micro-fluxmeter results at atmospheric pressure:

$T_{\mathrm{m}}=\frac{1}{2}\left(T_{\mathrm{f}}+T_{\mathrm{c}}\right), T_{\mathrm{r}}=\left[\frac{1}{4}\left(T_{\mathrm{c}}^{2}+T_{\mathrm{f}}^{2}\right)\left(T_{\mathrm{c}}+T_{\mathrm{f}}\right)\right]^{-1 / 3}$ (Büttner et al 1985), and $\rho=0.160 \pm 0.010 \mathrm{~g} \mathrm{~cm}^{-3}$.

\begin{tabular}{lllllll}
\hline Sample set & $e / \mathrm{mm}$ & $T_{\mathrm{c}} / \mathrm{K}$ & $T_{\mathrm{f}} / \mathrm{K}$ & $T_{\mathrm{m}} / \mathrm{K}$ & $T_{\mathrm{r}} / \mathrm{K}$ & $\lambda / 10^{-3} \mathrm{~W} \mathrm{~m}^{-1} \mathrm{~K}^{-1}$ \\
\hline SC5 & $11 \pm 0.1$ & $313 \pm 0.2$ & $287.5 \pm 0.2$ & $300.2 \pm 0.2$ & $300.4 \pm 0.2$ & $18.0 \pm 0.5$
\end{tabular}

Comparison of the results obtained for aerogel set SC5 shows that, as a first estimate, the three methods used in this project are quite equivalent to characterise such superinsulating material as silica aerogel. Indeed, the values obtained are quite closely centred around $0.019 \mathrm{~W} \mathrm{~m}^{-1} \mathrm{~K}^{-1}$.

To reinforce this experimental conclusion, a fourth technique was used to demonstrate the validity of the hot-band measurement. Thermal diffusivity estimated by this method was compared to the value obtained by the flash method. The results presented in table 4 clearly confirm the reliability of the hot-band measurements.

Table 4. Comparison of thermal diffusivity obtained by the hot-band and flash methods $\left(\rho=0.160 \pm 0.010 \mathrm{~g} \mathrm{~cm}^{-3}\right)$.

\begin{tabular}{lll}
\hline Sample set & $a_{\text {hot-band }} / 10^{-8} \mathrm{~m} \mathrm{~s}^{-2}$ & $a_{\text {flash }} / 10^{-8} \mathrm{~m} \mathrm{~s}^{-2}$ \\
\hline SC6 & $8.67 \pm 0.50$ & $9.28 \pm 0.50$ \\
SC7 & $8.98 \pm 0.50$ & $8.72 \pm 0.50$
\end{tabular}

It follows therefore that the popular hot-wire method can be used to measure the apparent thermal conductivity of silica aerogel. Furthermore, from a material and processing point of view, it can also be seen that the extraction process was repeatable, as shown by comparing the values for the $\mathrm{SC1}$ and $\mathrm{SC} 5$ aerogel sets. This demonstrates the reproducibility of the direct supercritical $\mathrm{CO}_{2}$ drying process in preparing panels with very low thermal conductivity. 


\subsection{Influence of direct radiative transfer at room temperature}

Some extinction coefficient measurements, carried out in parallel, confirmed for our samples the existence of the classic spectral transmission 'window' of silica aerogel located between 3 and $5 \mu \mathrm{m}$ (figure 5).

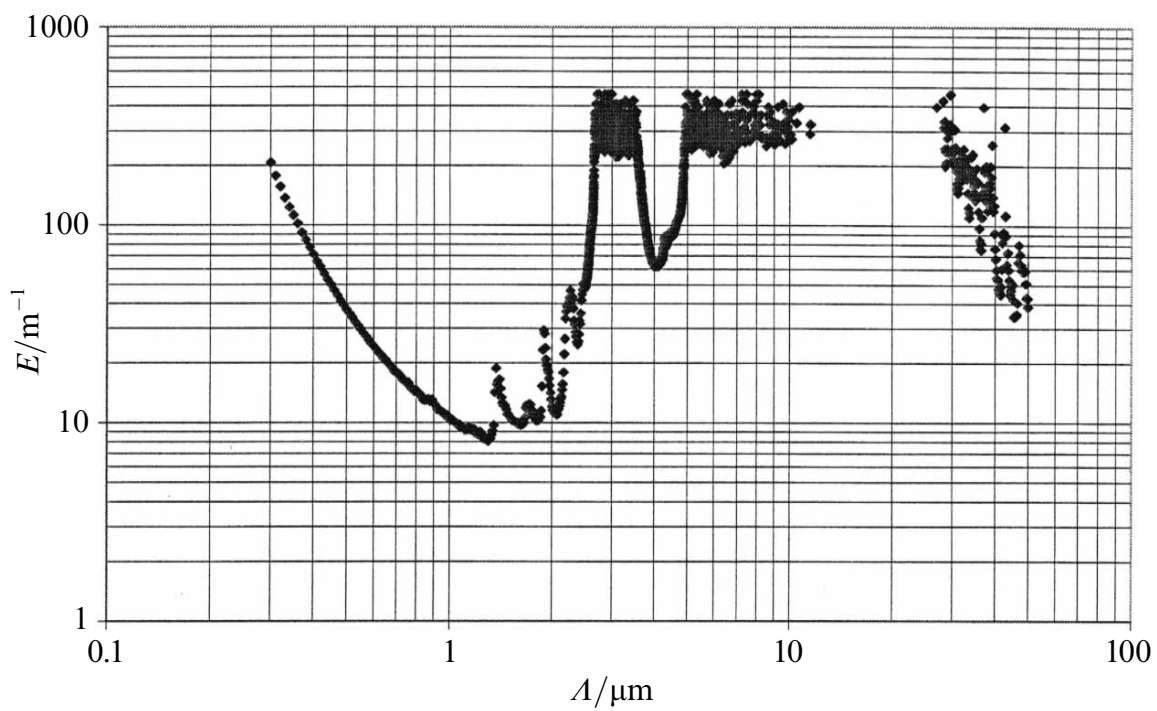

Figure 5. Extinction coefficient derived from normal transmission measurement $(\rho=0.160 \pm$ $\left.0.010 \mathrm{~g} \mathrm{~cm}^{-3}\right)$.

Having in mind the possible application of aerogels in double glazing, it appeared desirable to determine experimentally the influence of this low-extinction domain on the insulating performance of aerogel panels close to room temperature $(300 \mathrm{~K})$ and at temperature differences usually encountered in buildings $(20 \mathrm{~K})$. For this purpose, we used the micro-fluxmeter method, and we determined, at constant average temperature $(\sim 300 \mathrm{~K})$, the respective influences of aerogel thickness and the boundary surface emissivities. As shown in table 5, both decreasing the sample thickness $(e)$ and intercalating absorbing or reflecting thin film (with emissivity, respectively, superior to 0.95 and inferior to 0.05 ), between the aerogel and the fluxmeter, produced negligible thermal conductivity variations. So, in the first approximation, it can be concluded that at temperatures lower than $300 \mathrm{~K}$, the near-infrared transmission 'window' could have detrimental thermal influence in transparent building application field.

Table 5. Micro-fluxmeter measurements with $T_{\mathrm{f}}$ and $T_{\mathrm{c}}$, respectively, equal to $298 \mathrm{~K}$ and $313 \mathrm{~K}$ $\left(\rho=0.160 \pm 0.010 \mathrm{~g} \mathrm{~cm}^{-3}\right)$.

\begin{tabular}{llll}
\hline$e / \mathrm{mm}$ & $\begin{array}{l}\lambda / 10^{-3} \mathrm{~W} \mathrm{~m}^{-1} \mathrm{~K}^{-1} \\
\text { (without film) }\end{array}$ & $\begin{array}{l}\lambda / 10^{-3} \mathrm{~W} \mathrm{~m}^{-1} \mathrm{~K}^{-1} \\
\text { (with absorbing film) }\end{array}$ & $\begin{array}{l}\lambda / 10^{-3} \mathrm{~W} \mathrm{~m}^{-1} \mathrm{~K}^{-1} \\
\text { (with reflecting film) }\end{array}$ \\
\hline $2.5 \pm 0.05$ & $16.7 \pm 0.5$ & - & - \\
$3.8 \pm 0.05$ & $15.9 \pm 0.5$ & - & - \\
$4.0 \pm 0.1$ & $16.1 \pm 0.5$ & - & - \\
$6.0 \pm 0.1$ & $15.9 \pm 0.5$ & - & - \\
$8.0 \pm 0.1$ & $16.0 \pm 0.5$ & $16.0 \pm 0.5$ & $15.2 \pm 0.5$ \\
$12.0 \pm 0.1$ & $16.8 \pm 0.5$ & $16.8 \pm 0.5$ & $15.2 \pm 0.5$
\end{tabular}




\subsection{Optical characterisation}

With the double-glazing application still in our mind, we performed some optical transmission measurements with a Perkin Elmer $\lambda 19$ double-beam spectrophotometer (Rivero et al 1991). The percentage transmission (\%TR), used to characterise the transparency, was calculated from the following ratio in which $\tau_{\mathrm{v}}^{\text {nh }}$ and $\tau_{\mathrm{v}}^{\text {nd }}$ respectively represent the visible normal hemispherical and normal diffuse transmittance:

$$
\% \mathrm{TR}=\frac{\tau_{\mathrm{v}}^{\mathrm{nh}}-\tau_{\mathrm{v}}^{\mathrm{nd}}}{\tau_{\mathrm{v}}^{\mathrm{nh}}} .
$$

To take into account the Rayleigh scattering effects, the extinction coefficient $(E)$ at $550 \mathrm{~nm}$, the wavelength corresponding to the maximum eye sensitivity in the visible range, was also calculated:

$$
E=-\frac{1}{e} \log \left(\tau_{\mathrm{v}}^{\mathrm{nh}}-\tau_{\mathrm{v}}^{\mathrm{nd}}\right) .
$$

The results obtained for the typical aerogel set SC5 confirm the good optical properties of such silica aerogels. They showed that the percentage transmission is close to $90 \%$ while the extinction coefficient at $550 \mathrm{~nm}$ is $13 \mathrm{~m}^{-1}$.

\section{Conclusions}

Apparent thermal conductivity of aerogels derived from gels made with PEDS-Px silica precursor in ethylacetoacetate under hydrofluoric acid, and directly washed and dried with supercritical $\mathrm{CO}_{2}$, was measured by two transient methods (hot-wire and hot-band methods) and one steady-state technique (micro-fluxmeter method). A reproducibility study showed that these three methods give close values and can be considered as equivalent first-estimate tools.

In parallel, this study has shown that such monolithic silica aerogel panels provide a good thermal and optical compromise, and are very promising for transparent thermal insulation applications at room temperature.

Acknowledgements. ADEME (Agence de l'Environnement et de la Maîtrise de l'Énergie) is gratefully acknowledged for financial support. The authors thank all the members of the 'PACTE ADEME' for the collaborative work of which this study forms a part. The authors also specially thank Mrs A M Pardo (CSTB Grenoble) and Mr P Ilbizian (Centre d'Énergétique, École des Mines de Paris) for their respective help in optical measurements and sample preparation.

\section{References}

Batsale J C, Maillet D, Degiovanni A, 1994 Int. J. Heat Mass Transf. $37111-127$

Beck J F, 1977 J. Heat Transf. 99314

Biesmans G, Randall D, Français E, Perrut M, 1998 J. Non-Cryst. Solids 22536 -40

Büttner D, Caps R, Fricke J, 1985 High Temp. - High Press. 17375 -380

Carslaw H S, Jaeger J C, 1959 Conduction of Heat in Solids second edition (Oxford: Oxford University Press)

Degiovanni A, 1977 Rev. Gen. Therm. $185417-442$

Ebert H-P, Bock V, Nilsson O, Fricke J, 1993 High Temp. - High Press. 25391 - 402

Gobbe C, Bazin M, Gounot J, Dehay G, 1988 J. Polym. Sci. 26 857-864

Healy J J, De Groot J J, Kstin J, 1976 Physica C $82392-408$

Hrubesch L W, 1998 J. Non-Cryst. Solids $225335-342$

Ladevie B, 1998, Thesis, École des Mines d'Albi-Carmaux, Albi-Carmaux, France

Ladevie B, Batsale J C, Fudym O, 1999 Int. Commun. Heat Mass Transf. 27 473-484

Martin B, Durastanti J F, Peureux B, 1994 Entropie 147 47-52

Pajonk G M, Elaloui E, Achard P, Chevalier B, Chevalier J L, Durant M, 1995 J. Non-Cryst. Solids $1861-8$

Press W, Teukolsky S, Vetterling W, Flannery B, 1986 Numerical Recipes (Cambridge: Cambridge University Press)

Quenard D, Sallee H, 1991 Cahier du Centre Scientifique et Technique du Bâtiment No 2295

Rigacci A, 1998, Thesis, École des Mines de Paris, Paris, France 
Rivero J, Chevalier B, Chevalier J L, 1991 Cahier du Centre Scientifique et Technique du Bâtiment No 2522

Scherer G W, 1994 J. Sol-Gel Sci. Technol. $3127-139$

Schmidt M, Schwertfeger F, 1998 J. Non-Cryst. Solids $225364-368$

Tewari P H, Hunt A J, Lofftus K D, 1986, in Aerogels Ed. J Fricke (New York: Springer) pp $31-37$

Van Bommel M J, de Haan A B, 1994 J. Mater. Sci. 29 943-948

Wittwer V, 1992 J. Non-Cryst. Solids 145233 -236

Zeng S Q, Hunt A J, Cao W, Greif R, 1994 J. Heat Transf. 116 756-759 